PROCEEDINGS OF THE

AMERICAN MATHEMATICAL SOCIETY

Volume 126, Number 7, July 1998, Pages 2083-2088

S 0002-9939(98)04313-5

\title{
POINT-SPECTRUM-PRESERVING ELEMENTARY OPERATORS ON $B(H)$
}

\author{
QIN WANG AND JINCHUAN HOU
}

(Communicated by Palle E. T. Jorgensen)

\begin{abstract}
Let $H$ be an infinite dimensional separable Hilbert space over the complex field. Structure characterizations are given for some elementary operators on $B(H)$ which preserve point spectrum.
\end{abstract}

\section{INTRODUCTION}

Let $H$ be an infinite dimensional separable Hilbert space over complex field $\mathbf{C}$, and $B(H)$ the algebra of all bounded linear operators on $H$. For $T \in B(H)$, let $\sigma(T)$ and $\sigma_{p}(T)$ denote the spectrum and point spectrum of $T$ respectively. A linear map $\Phi$ on $B(H)$ is said to be spectrum-preserving (resp. point-spectrum-preserving) if $\sigma(\Phi(T))=\sigma(T)\left(\right.$ resp. $\left.\sigma_{p}(\Phi(T))=\sigma_{p}(T)\right)$ for all $T \in B(H)$. Jafarian and Sourour [5] proved that a spectrum-preserving linear surjective map $\Phi$ on $B(H)$ is either an automorphism or an anti-automorphism, that is, there exists an invertible operator $A \in B(H)$ such that $\Phi(T)=A T A^{-1}$ (an elementary operator of length 1!) or $\Phi(T)=A T^{t} A^{-1}$ for all $T$. In the last few decades, spectrum-preserving linear maps have been studied by many authors on matrix (or abstract) operator algebras (cf. [1], [2], [3], [5], [7] and the references in them). In fact, these are in the area of the so-called linear preserver problem which is currently an active research area in both matrix and operator theory [6].

Instead of spectrum-preserving maps, one can discuss the linear maps which preserve various parts of the spectrum, and sometimes obtain interesting results. If $\Phi$ is a linear surjective map on $B(H)$ which preserves point-spectrum (or surjectivityspectrum), P. Šemrl [7] proved that $\Phi$ must be an automorphism, never an antiautomorphism. If, in addition, $\Phi$ is positive, i.e., $\Phi(T)$ is a positive operator on $H$ whenever $T$ is, then one can easily deduce that $\Phi$ takes the form $\Phi(\cdot)=U(\cdot) U^{*}$ with a unitary operator $U \in B(H)$. Note that the assumption $\Phi$ is surjective is crucial for these results, as well as those in [5]. Without this assumption, $\Phi$ need not be of the forms mentioned above, as the map $\delta: B(H) \rightarrow B(H \oplus H)$, given by $\delta(T)=T \oplus T$ shows. So it is natural to ask if one can give a characterization for the structure of the non-surjective linear maps on $B(H)$ which preserve a subset of spectrum, in particular, point spectrum.

Received by the editors July 14, 1995 and, in revised form, March 15, 1996 and January 2, 1997.

1991 Mathematics Subject Classification. Primary 47A10, 47B47.

Key words and phrases. Hilbert space, elementary operator, point spectrum.

(C)1998 American Mathematical Society 
This question, however, seems very difficult to answer in general, and few results have been obtained so far. An important class of linear maps on $B(H)$ which contains a large number of non-surjective maps is the class of elementary operators. So we shall begin with the characterization for the elementary operators on $B(H)$ which preserve point-spectrum. Recall that a linear map $\Delta$ on $B(H)$ is called an elementary operator if there exist operators $A_{1}, A_{2}, \ldots, A_{n}$ and $B_{1}, B_{2}, \ldots, B_{n}$ such that $\Delta(T)=\sum_{i=1}^{n} A_{i} T B_{i}$ for all $T \in B(H)$. The positive integer $n=l(\Delta)=$ $\inf \left\{k: \Delta(\cdot)=\sum_{i=1}^{k} A_{i}(\cdot) B_{i}\right\}$ is called the length of $\Delta$. It is obvious that the above maps $\Phi(\cdot)=A(\cdot) A^{-1}$ and $\Phi(\cdot)=U(\cdot) U^{*}$ are unital elementary operators of length 1 and the latter is completely positive [8]. Since $H$ is of infinite dimension, one can identify $H$ and $H \oplus H$ with a unitary operator $\widetilde{U}=\left(U_{1} U_{2}\right) \in B(H \oplus H, H)$, where $U_{1}, U_{2} \in B(H)$. Thus the map $\delta$ mentioned above is in fact a completely positive unital elementary operator of length 2 , and takes the form $\delta(T)=\widetilde{U}(T \oplus T) \widetilde{U}^{*}=$ $U_{1} T U_{1}^{*}+U_{2} T U_{2}^{*}$. This observation motivates the following conjectures:

Conjecture 1. Let $\Delta$ be a completely positive unital elementary operator of length $n$ on $B(H)$. If $\Delta$ preserves point-spectrum, then there exists a unitary operator $\widetilde{U} \in B\left(H^{(n)}, H\right)$ such that

$$
\Delta(T)=\widetilde{U} T^{(n)} \widetilde{U}^{*}
$$

for all $T \in B(H)$. Here, $H^{(n)}=H \oplus \cdots \oplus H$, the direct sum of $n$ copies of $H$, and $T^{(n)}=T \oplus \cdots \oplus T \in B\left(H^{(n)}\right)$.

Conjecture 2. If $\Delta$ is a point-spectrum-preserving elementary operator of length $n$ on $B(H)$, then there exists an invertible operator $\widetilde{A} \in B\left(H^{(n)}, H\right)$ such that

$$
\Delta(T)=\widetilde{A} T^{(n)} \widetilde{A}^{-1}
$$

for all $T \in B(H)$.

Note that if a linear map $\Delta$ on $B(H)$ has the form of (2), then $\Delta$ is an injective endomorphism of $B(H)$ and $\Delta$ preserves point spectrum; if $\Delta$ has the form of (1), then $\Delta$ is an injective ${ }^{*}$-endomorphism and $\Delta$ is completely positive. For the case of $n=1$, it is easy to see both statements are valid. One can replace the pointspectrum in the conjectures by other subsets of spectrum and consider the analogous problems. We remark that the spectrum-preserving elementary operator of length 2 on $B(H)$ was considered by M. Gao [2] under the assumption $\operatorname{ran}\left(A_{1}\right) \cap \operatorname{ran}\left(A_{2}\right)=$ $\{0\}$.

In the present note, we shall give an affirmative answer to Conjecture 1. As for Conjecture 2, though we don't know whether the statement is true in general, we show that it is the case for the elementary operators of length 2 with the identity operator in its range. Moreover, this description reveals that the spectrumpreservability, the point-spectrum-preservability and the compression-spectrumpreservability are equivalent for those elementary operators under consideration.

Throughout this paper, we use $\operatorname{ker}(L), \operatorname{ran}(L)$ and $L^{*}$ to denote the kernel, the range and the adjoint of a linear operator $L$ respectively. Let $I$ be the identity operator on $H$ and $\langle\cdot, \cdot\rangle$ denote the inner product on $H$. For a linear manifold $M$ in $H, \bar{M}$ and $\operatorname{dim}(M)$ denote the closure and the dimension of $M$ respectively. The compression spectrum of $T \in B(H)$ is the set $\Gamma(T)=\{\lambda \in \mathbf{C}: \overline{\operatorname{ran}}(T-\lambda) \neq H\}$. 


\section{Results AND PROOFs}

Our first result is an affirmative answer to Conjecture 1.

Theorem 1. Let $\Delta$ be a completely positive unital elementary operator of length $n$ on $B(H)$. The following statements are equivalent:

(1) $\Delta$ preserves point-spectrum on $B(H)$.

(2) $\Delta$ preserves compression-spectrum on $B(H)$.

(3) $\Delta$ preserves spectrum on $B(H)$.

(4) There exists a unitary operator $\widetilde{U} \in B\left(H^{(n)}, H\right)$ such that $\Delta(T)=\widetilde{U} T^{(n)} \widetilde{U}^{*}$ for all $T \in B(H)$.

Hence, in either case, $\Delta$ is an injective ${ }^{*}$-endomorphism of $B(H)$.

Proof. It is obvious that (4) $\Rightarrow(1),(2)$ and (3).

$(1) \Rightarrow(4)$. For any non-zero vectors $x, y$ in $H$, let $x \otimes y$ denote the rank one operator defined by $(x \otimes y) z=\langle z, y\rangle x$ for $z \in H$. Since $\Delta$ is a completely positive elementary operator of length $n$ on $B(H)$, there exist linearly independent operators $D_{1}, D_{2}, \ldots, D_{n}$ in $B(H)$ such that $\Delta(\cdot)=D_{1}(\cdot) D_{1}^{*}+D_{2}(\cdot) D_{2}^{*}+\cdots+D_{n}(\cdot) D_{n}^{*}$ (see [3]). For any unit vector $x \in H, \Delta(x \otimes x)=D_{1} x \otimes D_{1} x+D_{2} x \otimes D_{2} x+\cdots+$ $D_{n} x \otimes D_{n} x$ is a positive operator of rank at most $n$. Since $\Delta$ preserves point spectrum, $\sigma_{p}(\Delta(x \otimes x))=\sigma_{p}(x \otimes x)=\{0,1\}$. Thus, $\Delta(x \otimes x)$ is the orthogonal projection of $H$ onto $\operatorname{span}\left\{D_{1} x, D_{2} x, \ldots, D_{n} x\right\}$. Similarly, for any two unit vectors $x, y \in H$ with $x \perp y, \Delta(x \otimes x+y \otimes y)=\Delta(x \otimes x)+\Delta(y \otimes y)$ is an orthogonal projection. Therefore, $\Delta(x \otimes x) \Delta(y \otimes y)=\Delta(y \otimes y) \Delta(x \otimes x)=0$, or equivalently, $\operatorname{span}\left\{D_{1} x, D_{2} x, \ldots, D_{n} x\right\} \perp \operatorname{span}\left\{D_{1} y, D_{2} y, \ldots, D_{n} y\right\}$. By a lemma in [3], we deduce that $D_{i}^{*} D_{j}=\alpha(i, j) I$ for some $\alpha(i, j) \in \mathbf{C}, i, j=1,2, \ldots, n$. So every operator in $\operatorname{span}\left\{D_{1}, D_{2}, \ldots, D_{n}\right\}$ is an isometry multiplied by a scalar.

Now suppose $x_{1}, x_{2}, \ldots, x_{n} \in H$ such that $D_{1} x_{1}+D_{2} x_{2}+\cdots+D_{n} x_{n}=0$. Left multiplying the equality by $D_{1}^{*}$, we have $x_{1} \in \operatorname{span}\left\{x_{2}, x_{3}, \ldots, x_{n}\right\}$ and there exist linearly independent operators $D_{2}^{\prime}, D_{3}^{\prime}, \ldots, D_{n}^{\prime} \in \operatorname{span}\left\{D_{1}, D_{2}, \ldots, D_{n}\right\}$ such that

$$
D_{2}^{\prime} x_{2}+D_{3}^{\prime} x_{3}+\cdots+D_{n}^{\prime} x_{n}=0 .
$$

By left multiplying the new equality by $\left(D_{2}^{\prime}\right)^{*}$ and continuing this trick, we will get $x_{1}=x_{2}=\cdots=x_{n}=0$, that is, the operator $\widetilde{U}:=\left(D_{1}, D_{2}, \ldots, D_{n}\right) \in B\left(H^{(n)}, H\right)$ is injective. Since $\Delta$ is unital, $\widetilde{U}$ is surjective and therefore invertible. So $\widetilde{U}^{*} \widetilde{U}=I$ and (4) holds.

Since $\sigma(F)=\sigma_{p}(F)$ for all finite-rank operators $F \in B(H),(3) \Rightarrow(4)$ follows from the proof of $(1) \Rightarrow(4)$. Note that $\sigma_{p}(T)=\Gamma\left(T^{*}\right)$ for all $T \in B(H)$; we have $(2) \Leftrightarrow(1)$. Now the proof is complete.

We are not able to answer Conjecture 2 in this note. But, for the case of $n=2$, we have the following results:

Theorem 2. Let $\Delta(\cdot)=A_{1}(\cdot) B_{1}+A_{2}(\cdot) B_{2}$ be an elementary operator of length 2 on $B(H)$ and $I \in \operatorname{ran}(\Delta)$. Then $\Delta$ preserves point-spectrum if and only if $\widetilde{A}:=$ $\left(A_{1}, A_{2}\right) \in B\left(H^{(2)}, H\right)$ is invertible and $\widetilde{B}:=\left(\begin{array}{l}B_{1} \\ B_{2}\end{array}\right)=\widetilde{A}^{-1}$, that is,

$$
\Delta(T)=\widetilde{A} T^{(2)} \widetilde{A}^{-1} \quad \text { for every } T \in B(H) .
$$

Thus $\Delta$ is an injective endomorphism of $B(H)$. 
Corollary 3. Let $\Delta(\cdot)=A_{1}(\cdot) B_{1}+A_{2}(\cdot) B_{2}$ be an elementary operator of length 2 on $B(H)$, and let $\Delta_{*}(\cdot)=B_{1}^{*}(\cdot) A_{1}^{*}+B_{2}^{*}(\cdot) A_{2}^{*}$. If $I \in \operatorname{ran}(\Delta)$, then the following statements are equivalent:

(1) $\Delta$ preserves point-spectrum on $B(H)$.

(2) $\Delta$ preserves compression-spectrum on $B(H)$.

(3) $\Delta_{*}$ preserves point-spectrum on $B(H)$.

(4) $\Delta_{*}$ preserves compression-spectrum on $B(H)$.

(5) $\Delta$ preserves spectrum on $B(H)$.

To prove Theorem 2, we need some lemmas. Lemma 1 is just an analogue of Lemma 1 in [5]. We omit its proof.

Lemma 1. Let $A \in B(H)$. Then $\sigma_{p}(T+A) \subset \sigma_{p}(T)$ for all $T \in B(H)$ if and only if $A=0$.

Lemma 2. Let $\Phi$ be a linear map on $B(H)$. Then

(1) If $\Phi$ is unital, i.e., $\Phi(I)=I$, then $\Phi$ preserves point-spectrum if and only if $\Phi$ preserves injections in both directions.

(2) If $\Phi$ preserves point-spectrum, then $\Phi$ is injective.

(3) If $\Phi$ preserves point-spectrum and $I \in \operatorname{ran}(\Phi)$, then $\Phi(I)=I$.

Proof. (1) is clear. (2) and (3) follow easily from Lemma 1.

In Lemmas 3 and 4 , let $\Delta(\cdot)=A_{1}(\cdot) B_{1}+A_{2}(\cdot) B_{2}$ be a unital elementary operator of length 2 on $B(H)$ which preserves point-spectrum. So $\left\{A_{1}, A_{2}\right\}$ and $\left\{B_{1}, B_{2}\right\}$ are linearly independent.

Lemma 3. $\operatorname{ker}\left(B_{1}\right) \cap \operatorname{ker}\left(B_{2}\right)=\{0\}$ and $\operatorname{ker}\left(A_{1}\right)=\operatorname{ker}\left(A_{2}\right)=\{0\}$.

Proof. Note that $\Delta$ preserves injections in both directions by Lemma 2; it is easy to see $\operatorname{ker}\left(B_{1}\right) \cap \operatorname{ker}\left(B_{2}\right)=\{0\}$. So we only need to prove the second statement.

We first show that at least one of $\operatorname{ker}\left(A_{1}\right)$ and $\operatorname{ker}\left(A_{2}\right)$ must be $\{0\}$. Since $\Delta$ preserves point-spectrum, $\Delta$ is injective by Lemma 2 . If $M:=\operatorname{ker}\left(A_{1}\right) \cap \operatorname{ker}\left(A_{2}\right) \neq$ $\{0\}$, let $P_{M}$ be the orthogonal projection of $H$ onto $M$. Then $P_{M} \neq 0$ and $\Delta\left(P_{M}\right)=$ 0 , a contradiction. Hence $\operatorname{ker}\left(A_{1}\right) \cap \operatorname{ker}\left(A_{2}\right)=\{0\}$.

If $\operatorname{ker}\left(A_{1}\right) \neq\{0\}$ and $\operatorname{ker}\left(A_{2}\right) \neq\{0\}$, take non-zero vectors $x_{1} \in \operatorname{ker}\left(A_{1}\right), x_{2} \in$ $\operatorname{ker}\left(A_{2}\right)$; then $x_{1}$ and $x_{2}$ are linearly independent. If for every $y \in H, B_{1} y$ and $B_{2} y$ are linearly dependent, then both $B_{1}$ and $B_{2}$ are rank-1 operators by a result in [3]. Thus, for any $T \in B(H)$ the rank of $\Delta(T)$ is at most 2 , contradicting the fact that $\Delta$ preserves injections. Hence there exists $y \in H, y \neq 0$ such that $B_{1} y$ and $B_{2} y$ are linearly independent. It is easy to find an injective operator $T \in B(H)$ such that $T B_{1} y=x_{1}, T B_{2} y=x_{2}$. But, in this case, $\Delta(T) y=A_{1} T B_{1} y+A_{2} T B_{2} y=A_{1} x_{1}+$ $A_{2} x_{2}=0$, again contradicting the fact that $\Delta$ preserves injections. Therefore, at least one of $\operatorname{ker}\left(A_{1}\right)$ and $\operatorname{ker}\left(A_{2}\right)$ must be $\{0\}$.

Without loss of generality, assume $\operatorname{ker}\left(A_{2}\right)=\{0\}$. We have to prove that $\operatorname{ker}\left(A_{1}\right)=\{0\}$. We will do this by considering two cases.

Case 1. $\operatorname{ran}\left(A_{1}\right) \cap \operatorname{ran}\left(A_{2}\right) \neq\{0\}$.

In this case, if $\operatorname{ker}\left(A_{1}\right) \neq\{0\}$, then there exist linearly independent $x$ and $y$ in $H$ such that $A_{1} x=A_{2} y$. Take $z \in H, z \neq 0$ such that $B_{1} z$ and $B_{2} z$ are linearly independent, and find an injection $T$ such that $T B_{1} z=x, T B_{2} z=-y$. Then $\Delta(T) z=0$, a contradiction. Hence $\operatorname{ker}\left(A_{1}\right)=\{0\}$. 
Case 2. $\operatorname{ran}\left(A_{1}\right) \cap \operatorname{ran}\left(A_{2}\right)=\{0\}$.

Suppose, on the contrary, that $\operatorname{ker}\left(A_{1}\right) \neq\{0\}$. Then

(a) $\operatorname{ker}\left(B_{2}\right)=\{0\}$. Otherwise, there exists $u \in \operatorname{ker}\left(B_{2}\right)$ with $u \neq 0$. Since $\operatorname{ker}\left(B_{1}\right) \cap \operatorname{ker}\left(B_{2}\right)=\{0\}$, we get $B_{1} u \neq 0$. Let $T$ be an injection such that $T B_{1} u \in$ $\operatorname{ker}\left(A_{1}\right)$; then $\Delta(T) u=0$, a contradiction. Hence $\operatorname{ker}\left(B_{2}\right)=\{0\}$.

(b) $A_{1} B_{1}$ is not a compact operator, in particular, $\operatorname{dim}\left(\operatorname{ker}\left(A_{1}\right)^{\perp}\right)=\infty$. To see this, note that $A_{2} B_{2}=I-A_{1} B_{1}$ (since $\left.\Delta(I)=I\right)$ and $\operatorname{ker}\left(A_{2} B_{2}\right)=\{0\}$. If $A_{1} B_{1}$ is compact, we have, by the Fredholm Alternative, that $\operatorname{ran}\left(A_{2} B_{2}\right)=\operatorname{ran}\left(I-A_{1} B_{1}\right)$ is closed and $\operatorname{dim}\left(\operatorname{ran}\left(A_{2} B_{2}\right)^{\perp}\right)=\operatorname{dim}\left(\operatorname{ker}\left(A_{2} B_{2}\right)\right)=0$. So $A_{2} B_{2}$ is invertible, contradicting the assumption $\operatorname{ran}\left(A_{1}\right) \cap \operatorname{ran}\left(A_{2}\right)=\{0\}$. Hence, $A_{1} B_{1}$ is not compact. In particular, $A_{1}^{*}$ is of infinite rank and $\operatorname{dim}\left(\operatorname{ker}\left(A_{1}\right)^{\perp}\right)=\operatorname{dim}\left(\overline{\operatorname{ran}}\left(A_{1}^{*}\right)\right)=\infty$.

We know that there exists $x \in H$ such that $B_{1} x$ and $B_{2} x$ are linearly independent. It is clear that $x \neq 0$ and $B_{2} x \neq 0$. Let $M=\left(\operatorname{span}\left\{B_{2} x\right\}\right)^{\perp}$. Since $\operatorname{dim}(M)=\operatorname{dim}\left(\operatorname{ker} A_{1}\right)^{\perp}=\infty$, there exists a unitary operator $U$ from $M$ onto $\left(\operatorname{ker} A_{1}\right)^{\perp}$. Define $T: H \rightarrow H$ by

$$
\left.T\right|_{M}=U, \quad T B_{2} x=0 .
$$

Then $T \in B(H), \operatorname{ran}(T)=\operatorname{ker}\left(A_{1}\right)^{\perp}$ and $\operatorname{ker}(T)=\operatorname{span}\left\{B_{2} x\right\}$. We will see that $\Delta(T)$ is injective, which contradicts the fact that $T$ is not injective and that $\Delta$ preserves injections in both directions. As a result, $\operatorname{ker}\left(A_{1}\right)$ must be $\{0\}$.

If $\Delta(T) h=0$ for some $h \in H$, then $A_{1} T B_{1} h=A_{2} T B_{2} h=0$ by the assumption $\operatorname{ran}\left(A_{1}\right) \cap \operatorname{ran}\left(A_{2}\right)=\{0\}$. Noting that $\operatorname{ran}(T)=\operatorname{ker}\left(A_{1}\right)^{\perp}$ and $\operatorname{ker}\left(A_{2}\right)=\{0\}$, we have $T B_{1} h=T B_{2} h=0$. Since $\operatorname{ker}(T)=\operatorname{span}\left\{B_{2} x\right\}$, there exist scalars $\lambda, \delta \in \mathbf{C}$ such that $B_{1} h=\lambda B_{2} x, B_{2} h=\delta B_{2} x$. Thus, together with $\operatorname{ker}\left(B_{2}\right)=\{0\}$, we get $h=\delta x$ and $\delta B_{1} x=\lambda B_{2} x$. But since $B_{1} x$ and $B_{2} x$ are linearly independent, we conclude that $\delta=\lambda=0$ and $h=\delta x=0$, that is, $\Delta(T)$ is injective. The proof is complete.

Lemma 4. (1) For every non-zero $x \in H, A_{1} x$ and $A_{2} x$ are linearly independent. (2) $\operatorname{ran}\left(A_{1}\right) \cap \operatorname{ran}\left(A_{2}\right)=\{0\}$.

Proof. (1) For any $A_{1}^{\prime}, A_{2}^{\prime} \in \operatorname{span}\left\{A_{1}, A_{2}\right\}$, if $A_{1}^{\prime}$ and $A_{2}^{\prime}$ are linearly independent, then there exist $B_{1}^{\prime}, B_{2}^{\prime} \in \operatorname{span}\left\{B_{1}, B_{2}\right\}$ such that

$$
\Delta(\cdot)=A_{1}^{\prime}(\cdot) B_{1}^{\prime}+A_{2}^{\prime}(\cdot) B_{2}^{\prime} .
$$

By Lemma 3, we have $\operatorname{ker}\left(A_{1}^{\prime}\right)=\operatorname{ker}\left(A_{2}^{\prime}\right)=\{0\}$. Hence, for any $\left(\alpha_{1}, \alpha_{2}\right) \neq(0,0)$, $\operatorname{ker}\left(\alpha_{1} A_{1}+\alpha_{2} A_{2}\right)=\{0\}$. Equivalently, for every non-zero $x \in H, A_{1} x$ and $A_{2} x$ are linearly independent.

(2) If $\operatorname{ran}\left(A_{1}\right) \cap \operatorname{ran}\left(A_{2}\right) \neq\{0\}$, then there exist $x, y \in H$ such that $A_{1} x=A_{2} y \neq$ 0 . By (1), we know that $x$ and $y$ are linearly independent. Take $z \in H$ such that $B_{1} z$ and $B_{2} z$ are linearly independent, and choose an injection $T$ such that $T B_{1} z=x$, $T B_{2} z=-y$. We again obtain a contradiction. Hence, $\operatorname{ran}\left(A_{1}\right) \cap \operatorname{ran}\left(A_{2}\right)=\{0\}$.

Proof of Theorem 2. Since $\sigma_{p}\left(T^{(2)}\right)=\sigma_{p}(T)$, there is nothing to prove for the sufficiency. Conversely, we know $\Delta(I)=I$ by Lemma 2 , so $\widetilde{A}:=\left(A_{1}, A_{2}\right)$ is surjective. By Lemmas 3 and $4, \widetilde{A}$ is injective, and the theorem follows immediately.

Proof of Corollary 3. Note that $\sigma_{p}(T)=\Gamma\left(T^{*}\right)$. It is easy to see that $\Delta$ preserves compression-spectrum if and only if $\Delta_{*}$ preserves point-spectrum. Now it is clear 
that (1)-(4) are equivalent by Theorem 2. The equivalency of (1) and (5) follows from Theorem 2 and a result in [4].

The authors wish to thank the referees for their helpful suggestions.

\section{REFERENCES}

1. B. Aupetit and H. du Tu. Mouton, Spectrum preserving linear mappings in Banach algebras, Studia Math., 109(1) (1994), 91-100. MR 95c:46070

2. Ming Chu Gao, Numerical range preserving linear maps and spectrum preserving elementary operators on $B(H)$, Chinese Ann. Math. Ser. A, 14 (1993), 295-301. MR 94g:47043

3. Jin Chuan Hou, Rank preserving linear maps on B(X), Sci. China Ser. A, 32 (1989), 929-940. MR 92b: 47052

4. Jin Chuan Hou, Spectrum preserving elementary operators on $B(H)$, preprint.

5. A. A. Jafarian and A. R. Sourour, Spectrum-preserving linear maps, J. Funct. Anal. 66 (1986), 255-261. MR 87m:47011

6. Chi-Kwong Li and Nam-Kiu Tsing, Linear preserver problems: A brief introduction and some techniques, Linear Alg. Appl., 162-164 (1992), 217-235. MR 93b:15003

7. P. Šemrl, Two characterizations of automorphisms on $B(X)$, Studia Mathematica, 105(2) (1993), 143-149. MR 94h:47064

8. M. Takesaki, Theory of Operator Algebras I, Springer-Verlag, New York, 1979. MR 81e:46038

Institute of Mathematics, Fudan University, Shanghai, 200433, People's Republic of CHINA

E-mail address: 970005@fudan.edu.cn

Department of Mathematics, Shanxi Teachers University, Linfen, Shanxi, 041004, People's Republic of China 